\title{
MUJERES Y DIVERSIDAD FUNCIONAL (DISCAPACIDAD): CONSTRUYENDO UN NUEVO DISCURSO
}

\author{
Carmen Mañas Viejo \\ Universidad de Alicante
}

\section{Introducción}

Las mujeres con discapacidad son cada día más conscientes de la múltiple discriminación que la sociedad les impone, y de las múltiples consecuencias que aquélla tiene para su desarrollo personal público y privado. Esa conciencia se plasma en la reivindicación de poder nombrarse a sí mismas, de sentirse referentes de ellas mismas. Reivindican sus voces a las teorías feministas y a las organizaciones sociales, y en poco tiempo han conseguido una verdadera transformación del concepto que las define. A partir de la clasificación internacional que en 1980 plantea la Organización Mundial de la Salud ${ }^{1}$, ante el

1. Deficiencia: «perdida o anormalidad de una estructura o función psicológica fisiológica o anatómica» que puede ser innata o adquirida, temporal o permanente, entre las que se incluye la existencia o aparición de una anomalía, defecto o perdida producida por un miembro, órgano, tejido u otra estructura del cuerpo, incluidos los sistemas propios de la función mental. La deficiencia representa la exteriorización de un estado patológico y, en principio, refleja perturbaciones a nivel del órgano.

Las deficiencias pueden catalogarse en tres grupos, si consideramos la salud como el estado de bienestar biológico, psíquico y social (OMS):

1) Fundamentalmente biológicas. Físicas, sensoriales y de carácter orgánico.

2) Fundamentalmente psicológicas. Deficiencia mental, trastornos de la conducta o de la personalidad.

3) Fundamentalmente socioculturales. La pobreza, la marginación y otros déficit sociales. En realidad cualquier clasificación incluye las tres dimensiones, ya que en las personas se da lo biopsicosocial, hecho multifacético, que es la discapacidad.

Discapacidad: «La ausencia debida a una deficiencia, de la capacidad de realizar una actividad en la forma o dentro del marco que se considere normal para un ser humano». Puede ser de conducta, comunicación, cuidado personal, locomoción o destreza. La discapacidad puede surgir como consecuencia directa de la deficiencia, pero ésta a veces no producen ninguna discapacidad (hay mas deficiencias que discapacidades).

Feminismo/s 13, junio 2009, pp. 9-20 
caos conceptual, los términos discapacidad, minusvalía y deficiencia, incorporan en su definición las repercusiones en la inserción social de las personas. El hegemónico modelo médico que ve a las personas con discapacidad como incompletas, alejadas de la norma y susceptibles de cura, se ve superado por lo que se conoce como el modelo social. Un modelo más completo que no culpabiliza a la persona con discapacidad y que entiende la discapacidad como una compleja colección de condiciones, muchas de ellas ambientales y de responsabilidad social. Con el modelo social, el concepto de normalidad se tambalea y se refuerza la adaptación social. Pero ni el modelo médico ni el social tienen en cuenta la especificidad de las mujeres, de forma que es el modelo crítico social el que a finales del siglo xx y principios del xxi integra y acentúa las experiencias específicas de ellas, que quieren y pueden salir de la invisibilidad.

El binomio mujer y discapacidad es una cuestión ideológica que no tiene agenda política hasta 1990 en el Seminario de Expertos de las Naciones Unidas sobre Mujeres con Discapacidad celebrado en Viena. Su repercusión se proyecta en la Cuarta Conferencia Mundial sobre Mujer en Beijing (1995), donde los gobiernos se comprometen a cumplir las previsiones que se han realizado a favor de las mujeres con discapacidad, lo que significa que se han comprometido a hacer lo necesario para que las recomendaciones que afectan a las mujeres con discapacidad se hagan realidad. En España en 2003 se celebra el I Congreso Internacional «Mujer y Discapacidad» en Valencia, donde se analizaron los problemas reales de las mujeres con discapacidad, con el objetivo de crear propuestas para la integración e igualdad de oportunidades en las diferentes dimensiones de la vida: económica, social, educativa, sanitaria, política e institucional, sexual, física y corporal. Y en 2005 la asociación « El Foro de Vida Independiente» aboga por un nombre menos discriminativo que discapacidad: diversidad funcional. El debate sigue y este trabajo quiere ofrecer una ventana abierta a la comunicación y al discurso de las mujeres con situación discapacitante, con diversidad funcional.

Verónica Grunewald Condori abre este número con su trabajo «Inflexiones de la vida: un par de lecciones», en el que narra en primera persona su experiencia con una situación discapacitante que le sobreviene en forma de enfermedad neurodegenerativa, como ella misma dice, de nombre extraño, antes de cumplir los 40. El discurso de Verónica es singular, propio, y representa la

Minusvalía: «una situación desventajosa para una persona, consecuencia social de una deficiencia o de una discapacidad, que limita o impide el desempeño de un rol que es normal en su grupo. Las minusvalías pueden ser de orientación, de independencia física, de movilidad, ocupacionales, de integración social o de autosuficiencia económica». 
pluralidad de las mujeres que se posicionan contra la resignación y el conformismo, y deciden seguir estando vivas. No es un discurso de víctimas ni de heroínas, es una historia de vida que diferencia el ser del estar, que se esfuerza por seguir siendo ella misma, tratando de adaptarse a su nueva situación discapacitante, a su diversidad funcional, buscando en su interior la forma de afrontar su enfermedad sin dejarse arrollar por ella. Sorteando los obstáculos físicos, psicológicos, cognitivos y sociales, que se han hecho cotidianos, transformando las energías que encuentra en su camino en las herramientas con las que construye sus barricadas contra la enfermedad. La autora es madre y se pregunta quién está preparado para cuidar a una madre, y no hay respuesta, pero no deja espacio a la inmovilidad mental. Su narrativa se asienta en la filosofía existencial del ser y su lucha es una nueva forma de estar. Un estar fuera de toda norma y no por ello deja de sentirse femenina, plural, absorbente y díscola porque ha desobedecido las normas y se ha rebelado contra el destino de una enfermad con creatividad.

El siguiente trabajo pone de manifiesto la necesidad de una profunda reflexión sobre la discapacidad y el género. Laura Viñuela Suárez en «Mujeres con discapacidad: un reto para la teoría feminista» aborda con rigor, claridad y valentía el discurrir de las teorías feministas y las de la discapacidad, cómo ambas se han ido haciendo hueco en un lento proceso de deconstrucción, precedido por movimientos sociales previos. Para ambos campos, la discapacidad y los estudios de género, la década de los 70 y 80 fueron decisivas para su consolidación en el ámbito académico, y sin embargo no será hasta la década de los 90 cuando ambos se unan, ya que hasta entonces ni los estudios sobre discapacidad habían tenido en cuenta a las mujeres, ni los estudios sobre las mujeres habían tenido en cuenta a la discapacidad.

Uno de los puntos de encuentro entre ambas teorías es el que hace referencia al cuerpo y sus conceptualizaciones, basadas, fundamentalmente, en la idea cartesiana que establece la separación entre la mente y el cuerpo. La identidad de la mujer queda atrapada en un cuerpo que la discapacita para dominar y ser capaz, y el cuerpo es también quien atrapa a la persona con discapacidad haciendo invisible cualquier otra cualidad no corpórea.

El cuestionamiento de la idea cartesiana que defiende la dualidad entre el cuerpo y la mente es la clave de un cambio de modelo, de paradigma, es el comienzo del camino del modelo social. Un modelo que no pretende ser excluyente pero que enfatiza en los presupuestos ideológicos que subyacen a toda terminología y considera las consecuencias sociales de la enfermedad, porque son ellas las que perturban la vida diaria de las personas que las tienen. 
A través de la sujeción de las personas a sus cuerpos, la ideología dominante ha conseguido hacer de la sexualidad y de la maternidad la conceptualización de la mujer, y las mujeres con discapacidad han quedado excluidas y consideradas asexuadas y sin belleza, parámetros que ellas reivindican. Esta reivindicación se presenta como un reto a superar en la identidad de las mujeres, que, para empoderarse, en opinión de la autora, quizás deba pasar del análisis sexo-género al cuestionamiento de la propia noción de mujer.

Siguiendo dentro de este mismo análisis teórico de los estudios de mujeres y discapacidad, el trabajo presentado por Miriam Arenas Conejo, "Las mujeres con diversidad funcional (discapacidad) como agentes de transformación social: Una perspectiva internacional», defiende la hipótesis de que las mujeres en este comienzo de siglo están adquiriendo un nuevo rol protagonista en los procesos de transformación social, considera que las mujeres son promotoras y facilitadoras activas de transformación social.

Este artículo presenta los resultados descriptivos de una investigación cuyo objetivo es aproximarse, ver de cerca a las mujeres con diversidad funcional. Es un proyecto global al amparo de Naciones Unidas, que comienza con un breve recorrido del nacimiento, en las mujeres con discapacidad, de la conciencia de tener voz propia, de cómo comienzan a ser consideradas y a considerarse públicamente como agentes de transformación social.

La investigación parte del análisis de movimientos sociales caracterizados por compartir la creencia de que las actitudes existentes en la sociedad hacia el valor humano, económico y social de las mujeres con diversidad funcional son erróneas e injustificadamente negativas. Y da cuenta de las estrategias defensivas y pro-activas que los movimientos de mujeres con diversidad funcional están poniendo en marcha en catorce países. La estrategia defensiva más importante y global pasa por la consideración de la discapacidad o diversidad funcional desde la perspectiva de los derechos humanos y la lucha por la eliminación de las barreras discapacitantes. En cuanto a las estrategias pro-activas, van mucho más allá del género y la diversidad funcional, se encaminan hacía una flexibilidad de los conceptos de feminidad y discapacidad que pasa por la radicalización de la democracia, es decir, por una ciudadanía activa que ponga el acento en las capacidades universales de las mujeres diversas que tanto nos asemejan y no en las que nos diferencian.

Y buscando semejanzas, Victoria Robles Sanjuán en «La extraña relación entre 'la incapacidad de' y la 'sobrecapacidad para' en la literatura escolar para niñas y mujeres (siglos XIX $y \mathrm{xx}$ )», reflexiona sobre la paradoja, nada inocente, de las representaciones del cuerpo de las mujeres y de sus significados en los 
textos dirigidos a niñas, maestras y madres editados en el último tercio del siglo xix español y primero del siglo xx.

La moral y el cuerpo de las mujeres son la clave de sus capacidades e incapacidades. En los textos estudiados se encuentran abundantes ejemplos sobre cómo la higiene física y doméstica está íntimamente ligada con la buena conducta moral. El cuerpo físico de la mujer queda invisibilizado en favor del cuerpo ajeno, el de los que están bajo su responsabilidad. Estará presente en cuanto a los deberes que tienen que cumplir pero invisible como entidad. El cuerpo de la mujer es todo moral. Todo lo que es antihigiénico y antieconómico es para la mujer amoral. Un cuerpo sobrecapacitado para cuidar a los demás e incapacitado para gobernarse a sí mismo. Un cuerpo que además debe satisfacer las miradas externas, y para ellas serán los adornos pero prontamente son advertidas del peligro que encierra su belleza, su cuerpo, si no lo gobiernan. Las diversiones y entretenimientos apenas ocupan espacio y nunca en la intimidad, siempre bajo la atenta mirada de los padres primero y del marido después. Y del cuerpo invisible a la maternidad y de la maternidad a la niña de los cuentos, a la que hay que imitar, inerte, preciosa, obediente y dechado de virtudes perpetuamente infantiles, que las representan como inválidas mentales y físicas y sin embargo ello no las incapacita para las mil y una tareas del hogar, que han de realizar sin descanso, sin queja y con alegría. Pagando así el tributo de ser niñas, de ser mujeres sin cuerpo, y sucediéndose una a la otra.

En línea con esta reflexión, el trabajo de Esther López Zafra y Rocío García-Retamero, titulado «Mujeres y liderazgo: discapacitadas para ejercer el liderazgo en el ámbito público», se centra en considerar que la discapacidad o incapacidad de la mujer en posiciones de liderazgo proviene más del entorno social que la rodea y de las inferencias enraizadas en el sistema cognitivo de las personas, que en una incapacidad o discapacidad real para ejercer el liderazgo.

Tanto para las mujeres con discapacidad o sin ella, han sido y son necesarias la promulgación de leyes que arbitren la igualdad de oportunidades, y en ambos casos la igualdad real está lejos, pese al gran avance conseguido, el cual no hay que desvalorizar en absoluto. De su análisis sobre la situación real de las mujeres en la sociedad actual, concluyen que todavía existen y están en valor prejuicios y atribuciones que les impiden a ellas mismas creer en la igualdad real, lo que pone en evidencia que el avance legislativo no ha podido modificar la asunción y atribución de roles desigualmente valorados. Una prueba de ello es la ratio de mujeres desempleadas frente a los hombres, una remuneración económica entre un 25 y un 30 por ciento menor que el de 
los varones y una dedicación tres veces mayor a las tareas del hogar en ellas que en ellos. La elección de estudios universitarios es otra brecha abierta en el género, los estudios técnico superiores siguen teniendo una escasa representación femenina, mientras que en las humanidades, las ciencias experimentales y de la salud son ellas mayoría. Y si nos centramos en las posiciones de poder, en los puestos de toma de decisión en los distintos ámbitos, vemos que la brecha del género sigue latiendo con fuerza. Por ejemplo, en la Universidad sólo un $14 \%$ de los puestos de responsabilidad están ocupados por mujeres, y eso pese a que numéricamente es mucho mayor el número de mujeres que de hombres. En el sector de las finanzas no llegan a un 3\% las mujeres en puestos de liderazgo y sube un poquito más en la política, hasta el 29\%. Pero además, estas mujeres que ocupan puestos de liderazgo, deben enfrentarse a dos juicios: el primero tiene que ver con el cómo y por qué han llegado, es decir, representan cuota, representan subordinación afectiva, representan valía; el segundo juicio que deben de pasar es el análisis de su tipo de liderazgo: femenino o masculino. Hay teorías para todos los gustos, hay teorías que defienden que el liderazgo no tiene género y teorías que defienden lo contrario y que polarizan el liderazgo de las mujeres en masculino y conservador, o femenino y trasformador. De hecho, los trabajos revisados proporcionan datos sobre el hecho de que este prejuicio se debe fundamentalmente a la percepción de incongruencia entre las características estereotípicas asignadas al liderazgo (masculinas) y las propias de las mujeres (femeninas). Frente a esta situación, las autoras proponen una educación temprana en igualdad y la potenciación de la igualdad de oportunidades por parte de las instituciones, para que no se perciba esa diferencia tan grande entre los distintos roles y se evalúen las características de las personas independientemente de su sexo.

Al hilo de la importancia de la construcción y activación de los estereotipos de género Beatriz Montes Berges, en su artículo «Exposición a estereotipos y categorías de género: Consecuencias y evaluaciones", nos aporta un interesante estudio de corte empírico, sobre la activación de categorías mediante priming subliminal. En él se parte de la hipótesis de que en las tareas de rendimiento verbal y matemático se prevé un efecto principal de la condición priming-no priming, ya que la activación de los estereotipos conllevaría una mejor realización de las tareas que implican habilidades propias de cada género. La elección de las tareas tiene que ver con su asignación tradicional a estereotipos de género. Justificando su elección en el hecho de que aunque todos los estudios apuntan que el rendimiento de hombres y mujeres en este tipo de tareas es similar, cuando se encuentran diferencias se asocian con el efecto de la amenaza del estereotipo.

Feminismo/s 13, junio 2009, pp. 9-20 
Los resultados obtenidos invitan a deducir, en línea con muchas otras investigaciones de corte cognitivo social, que la presentación de las categorías activaba los estereotipos relacionados con ellas, haciéndolos más accesibles, de manera que cuando se presentaban los estereotipos se necesitaba menos tiempo para procesarlos y dar una respuesta según éstos. Además se proponía observar si estas activaciones influían en el rendimiento de las tareas verbales y matemáticas, de manera que los participantes se comportaran según los estereotipos: los hombres mejores en la prueba matemática y las mujeres mejores en la tarea verbal. Así, en la tarea verbal se encuentra, tal y como se anticipaba, que cuando los participantes tuvieran activadas las categorías de género por la presentación subliminal, las mujeres puntuarían más y los hombres menos conforme a sus estereotipos. Sin embargo, en la tarea matemática, ocurría lo contrario: cuando los participantes no han realizado la tarea de priming, se encuentra que los hombres puntúan más y las mujeres menos. Estos resultados, en contra de la hipótesis de partida, los explica la autora argumentando que quizás puedan deberse a que los estereotipos sobre las habilidades especiales de cada género están cambiando, percibiéndose cada vez más a las mujeres como buenas en tareas matemáticas y/o científicas, tradicionalmente consideradas masculinas.

Es interesante observar que los estudios muestran que cuando se mide el rendimiento verbal o matemático de hombres y mujeres sin que sean expuestos a expectativas o estereotipos previos, no se encuentran diferencias.

De lo que no cabe duda es de que tanto las estructuras sociales que nos envuelven como la cultura en la que crecemos influyen directamente en la construcción y moldeamiento de la identidad. Grace Shum y Ángeles Conde, en su artículo «Género y discapacidad como moduladores de la identidad», parten de la concepción cartesiana donde lo femenino se asimila a lo privado y lo masculino a lo público, configuran también las relaciones de poder entre lo femenino y lo masculino, y nos presentan una descripción de las representaciones del cuerpo que un grupo diverso de 78 mujeres tiene. Y de qué forma el tipo de discapacidad física, sensorial, sobrevenida o congénita, el grado de minusvalía, el nivel de estudios y la edad son variables que modulan las diferencias de la autopercepción de la imagen corporal. La corporeidad del ser se desarrolla en un proceso de socialización que implica una interiorización de valores y creencias del grupo de referencia, que nos posibilita la capacidad de diferenciarnos las unas de las otras, articulándose la intersubjetividad cultural como potente constructor de la identidad.

Los parámetros de referencia con los que han de medirse las mujeres son fundamentalmente la belleza y la salud, ambas dedicadas al deleite y cuidado 
de los demás, nunca a sí mismas. En estos parámetros las personas, mujeres con discapacidad, no caben, están fuera, sin embargo son estos parámetros los que representan la verdadera discapacidad. Los parámetros que excluyen a las mujeres con discapacidad las convierten en asexuadas, eternamente infantiles e improductivas, pese a que en muchos casos llevan la carga y cuidado del hogar igual que cualquier otra mujer. La infatilización consigue la negación de las necesidades afectivo-sexuales que como personas completas tienen, y la improductividad fomenta, entre otros, el sentimiento de incompetencia maternal. Pero esta visión tan negativa no es general. Hoy las mujeres con discapacidad tienen voz y han conseguido existir, es decir, estar en la agenda política, y esto fundamentalmente gracias a su propia mentalización, a creerse personas plenas y con plenos derechos, que tienen como objetivo crecer, desarrollarse y ser autónomas en las elecciones de cada día. Ese es el camino, ahondar en la mentalización que ellas revindican más allá de medidas generales y concretas. La discapacidad, sin lugar a dudas, es una variable que interviene en la construcción de la identidad tanto de hombres como de mujeres. Sin embargo el modo en el que lo hace es diferente, debido a la gran diferencia que existe en el significado social que tan distinto es para hombres y mujeres con y sin discapacidad.

Ahondando en el estudio de la discapacidad y el género, Asunción Moya Maya nos presenta su artículo "La sexualidad en mujeres con discapacidad. Perfil de su doble discriminación», sin duda un tema aún hoy tabú. La autora parte en su artículo de considerar la discapacidad en las mujeres como un elemento más discriminador y negativo que en los hombres. Comienza contextualizando la situación de la mujer con discapacidad que describe como la minoría mayor, y enfatiza su escaso poder de decisión en el ámbito familiar y laboral y su escasa o nula participación social, lo que conlleva menor formación y en consecuencia menores oportunidades, señalando que la mayor diferencia se abre en el campo de la dependencia, donde mas del 65.4 por ciento del total de las personas con algún grado de dependencia son mujeres. La feminización de la pobreza es el boceto lógico que representan las mujeres con discapacidad. Contextualizado el perfil de las mujeres con discapacidad se centra en la vida afectiva sexual de éstas, que en su opinión aún hoy sigue estando relegada en los estudios e incluso en sus propios discursos. La mayoría de los esfuerzos se centran en la formación y en la accesibilidad al mundo laboral, pero sus necesidades afectivo-sexuales se siguen negando y en consecuencia siguen sin atenderse. Falta un largo camino por recorrer en el reconocimiento de sus derechos sexuales y reproductivos. Toda persona sana que se acerque a una mujer con discapacidad despierta recelo, pero si es con

Feminismo/s 13, junio 2009, pp. 9-20 
carácter afectivo-sexual se convierte en sospechosa. Las dificultades de acceso a una vida de relación normalizada (dificultad de acceso a bares, discotecas y en general lugares de ocio para jóvenes) las aísla, y esa vivencia se instaura en el núcleo de su identidad como baja autoestima. El cuerpo de las mujeres con discapacidad se percibe tan vulnerable que se desea invisible. Pero las mujeres con discapacidad se hacen mayores y reivindican su derecho a la sexualidad, a la información, a la planificación familiar, a tomar el control de sus vidas, de su sexualidad y de su salud reproductiva.

No hay toma de control sin autonomía económica y ésta está fuera del alcance de muchas mujeres. En el siguiente trabajo, "Situación laboral de las mujeres con discapacidad física y sensorial en la provincia de Alicante», realizado por quien escribe estas líneas junto a Almudena Iniesta Martínez, tratamos de describir la situación laboral de las mujeres con discapacidad física y sensorial de la provincia de Alicante, tanto en el ámbito privado como en el público. Queremos aproximarnos a cómo ellas viven su integración en el medio laboral. Para ello recogemos mediante entrevistas en profundidad con un formato semiestructurado sus opiniones y reflexiones sobre su situación laboral y sobre sus expectativas. El análisis exhaustivo de las narrativas de las $50 \mathrm{mu}$ jeres que participan activa y voluntariamente en la investigación, las interpretamos mediante la elaboración de categorías demográficas y descriptivas de las unidades significativas de sus historias de vida. Dentro del ámbito laboral hemos constatado que en sus discursos coinciden en percibirse relegadas al desempeño de trabajo no mercantilizado, es decir, poco o nada remunerado. En esta percepción, casi colectiva, confluyen la atribución del rol femenino y su escasa formación. En su mayoría tienen trabajos feminizados, que equivale a decir poco reconocidos socialmente y poco remunerados económicamente. Son conscientes de que la percepción social que se tiene de ellas es negativa y deriva del desconocimiento, se les considera poco productivas, dependientes y de escaso potencial. La consecuencia lógica de esta percepción es un etiquetaje, en muchas ocasiones insalvable para la obtención de un empleo. El desconocimiento que sobre la discapacidad tiene la sociedad impide que se les proporcionen las ayudas técnicas que necesitan para desarrollar una actividad en igualdad de condiciones. Además son conscientes de que el trabajo al que pueden acceder está muy condicionado por el hecho de ser mujeres y por el tipo de discapacidad. Por tanto son conscientes de la discriminación de género que soportan. Aunque la asumen, en su mayoría, con resignación, son cada vez más las mujeres que se rebelan contra esta estigmatización, y comienzan a reivindicar formación y adaptaciones para renegociar democráticamente su lugar en el mercado laboral.

Feminismo/s 13, junio 2009, pp. 9-20 
En relación a las ayudas técnicas, el trabajo de Elvira Ramos García titulado: "Autonomía personal de las mujeres con discapacidad: el caso de la accesibilidad a las ayudas técnicas y nuevas tecnologías», se centra en el asesoramiento para la autonomía personal de las mujeres con discapacidad, con el fin de realizar propuestas y de agilizar la solución de los déficits que en esta materia se están produciendo. Elvira Ramos justifica su trabajo dentro del marco legal nacional e internacional vigente, del cual se deduce que las ayudas técnicas son, en este momento, consideradas herramientas fundamentales para el logro de la vida independiente y la integración social de las mujeres con discapacidad, y por tanto deben constituir un derecho subjetivo y garantizado por ley. Los déficits a superar son por un lado la falta de información, no sólo de las mujeres con discapacidad sino también -y esto es más preocupante aún, por sus implicaciones-, la falta de información y formación de los profesionales que han de asesorarlas. Y por otra parte, un segundo déficit es el excesivo precio y mala distribución sectorial, que hace de estas herramientas fundamentales para el desarrollo personal y profesional productos de lujo. Propone una mayor financiación pública y sobre todo una mayor participación en todo el proceso del desarrollo, elaboración y puesta en servicio de estas herramientas de las propias mujeres, que en definitiva son las usuarias y exigen el poder aportar su experiencia y conocimiento.

Abordamos a continuación la discapacidad intelectual, y para ello contamos con el trabajo: «Contribución a una crítica epistemológica de la discapacidad intelectual» realizado por María Cecilia Tamburrino. Basado en una investigación etnográfica que la autora y su equipo tienen en curso en la actualidad en un hospital psiquiátrico, con pacientes crónicos, es decir, institucionalizados más de 20 años, del área metropolitana de Buenos Aires. Este trabajo pone en cuestión el valor objetivo del diagnóstico, argumentando que él mismo no es sino una técnica política que involucra consideraciones morales y epistémicas. Comienza por revisar su definición, tanto en la literatura científica como a través del discurso de los y las profesionales (psiquiatras, psicólogos y neurólogos) que trabajan en la actualidad en el campo clínico del retraso mental, hallando una apabullante confluencia en la terminología, tanta que justifica su escaso interés. En el núcleo de la definición del retraso mental está lo que no hay, bien por causa genética, biológica, o bien por causa contextual dialéctica. Tanto el diagnóstico como el pronóstico están excesivamente claros, el retraso mental es una falta de capacidad que afecta a la esfera de la intelectualidad y su pronóstico es malo, no se cura y cursa con el paciente a lo largo de toda su vida. La falta de inteligencia está unida a la falta de razonamiento abstracto y a los juicios de valor. Al estar afectada la inteligencia está afectada

Feminismo/s 13, junio 2009, pp. 9-20 
también su capacidad de realizar juicios sobre la realidad concreta y por tanto su afectividad y su actividad, donde predomina la impulsividad, el primitivismo, la simpleza, el bajo control de sí mismo... al no contar con los recursos necesarios para el autodominio, disminuida su voluntad y su capacidad de juicio, las personas con retraso mental quedan destinadas a una situación de heteronomía y dependencia: impedidos moralmente. Y su prescripción médica recomienda la institucionalización con el fin de darles aquello que les falta. Esta unanimidad se rompe frente al concepto de la inteligencia y la forma de medirla. Para unos es la capacidad de razonar de forma abstracta, para otras la capacidad de adaptarse, para otros la capacidad de dar respuestas nuevas, de crear, para otras las inteligencias son múltiples y así podríamos seguir añadiendo tantas definiciones de qué es la inteligencia y cómo medirla como autores y profesionales analizáramos. La inteligencia delimita campos epistémicos, por un lado, el apego a la realidad concreta, sensible e intuitiva, como la maternidad y por el otro, lo abstracto, lo sublime, la realidad conceptual. Tamburrino argumenta, a través de los discursos de los y las profesionales que han intervenido en su investigación, cómo las mujeres han sido definidas como seres con déficit intelectual, dependientes e incapaces de juicio autónomo y discriminativo, lo que ha justificado su exclusión de la vida pública, de la formación y de la política. Consideradas incapaces de crear, condenadas a la imitación, su déficit intelectual y moral es intrínseco a su sexo por naturaleza. La falta de inteligencia despoja a los seres humanos de grados de humanidad, convirtiéndola en un operador político que atraviesa las categorías de género, raza, etnia y clase social. La conclusión, a nuestro juicio, más interesante que señala el artículo es que la inteligencia ha servido para construir un discurso sobre la desigualdad en general y sobre la desigualdad de género en particular, y además para validarlo. Y son las teóricas feministas las primeras en desenmascarar los elementos que componen la inteligencia, sus procedimientos, sus técnicas y su saber, iluminando su politización.

Terminamos con una experiencia interesante y novedosa que pone en entredicho la autonomía que pueden alcanzar las personas con discapacidad intelectual. Marta Liesa Orus y Elias Vived Conte, en «La adquisición de competencias para la vida diaria: un estudio de casos. Diferencias de sexo entre personas con discapacidad», nos describen y analizan parte de los resultados obtenidos en el proyecto, que se está desarrollando en la ciudad de Huesca, al que han denominado «hacia una vida independiente», basado en experiencias similares realizadas en Barcelona y Murcia. Su idea eje es que la calidad de vida de una persona se puede medir por el nivel de elección que tiene. El objetivo de este programa, incluido dentro de los denominados de transición hacía una 
vida independiente, es que las personas con discapacidad intelectual aumenten su independencia y con ello mejoren su calidad de vida. Los servicios que se prestan a quienes participan voluntariamente en un programa de transición a la vida independiente dependen del grado de discapacidad o minusvalía que presentan. Se les ayuda a realizar las tareas del hogar, a cuidar la higiene personal o a saber planificar su tiempo libre, entre otras cosas.

Los resultados que nos presentan pertenecen al análisis de datos obtenidos durante el curso escolar 2007-2008. En ese curso, la vivienda utilizada para el proyecto está habitada por dos estudiantes de magisterio y dos personas con discapacidad: una chica y un chico, ambos con una minusvalía asociada a su discapacidad intelectual. Su trabajo pone de manifiesto que las personas con discapacidad tienen las mismas ganas de independencia que los chicos y chicas sin discapacidad, pero en sus familias se vive con mayor temor, es decir, sus expectativas son más pequeñas, viven en una burbuja de superprotección. Además se evidencia que las chicas vienen más preparadas en las tareas del hogar, propias de su rol, que ellos, lo que subraya, una vez más, que la variable género atraviesa las construcciones identitarias de todas las personas.

Por último, quiero aprovechar esta ocasión para agradecer a todas las personas que han colaborado en este número su esfuerzo, dedicación y amabilidad, pero sobre todo su compromiso personal con la igualdad de oportunidades.

Feminismo/s 13, junio 2009, pp. 9-20 